\title{
How to promote COVID-19 pandemic prevention and control: A game theory analysis
}

\author{
Jin Yan \\ China Economics and Management Academy, Central University of Finance and Economics, Beijing \\ 100081, China \\ E-mail:2019212149@email.cufe.edu.cn
}

\begin{abstract}
The outbreak of the COVID-19 (Corona Virus Disease 2019) has been becoming the most serious public health event in the world, threatening the health of hundreds of millions of people. The anti-pandemic policies mostly aim to prevent the spread at domestic and the import from abroad. In this paper, we discuss the issue of individual protection measures and customs quarantine strategy based on game theory. First, we develop a $2 * 2$ Bayesian model and show that individual protection decisions depend on the size of excess payoff of taking protective measures (EPP). EPP is affected by the protection cost, the protective efficiency, the risk exposure and the information disclosure in this model. Then, we describe a repeated game between customs and airlines, and discuss the ideal strategy for customs to prevent the virus input. It comes out that a punishment mechanism for an airline is meaningful to prevent the import of the virus only if the discount rate of the airline is greater than the threshold. The threshold is positively correlated with the detection cost, and it is negatively correlated with the revenue of the airline and the testing reliability. We can also benefit from establishing a credit mechanism to give airlines that deviate from cooperation an opportunity to re-cooperate, especially for those in low risk areas. This study provides theoretical support for some government policies to prevent COVID-19, and underscores the significance of considering individual and organizational decision-making in health policy making.
\end{abstract}

Keywords: COVID-19, pandemic, matrix games, game theory, protective behavior, virus import

\section{Introduction}

Since its discovery at the end of 2019, COVID-19 has spread all over the world, and more than 165 million people have been infected. The secretary of United Nations, António Guterres even regards the new coronavirus as "the biggest test we have faced since the establishment of the United Nations" [1]. The Asian Development Bank estimated that the global economic loss caused by the pandemic ranges from $\$ 5.8$ trillion to $\$ 8.8$ trillion, which is equivalent to $6.4 \%-9.7 \%$ of global GDP (gross domestic product). ${ }^{1}$ The prevention and control of the pandemic have been becoming the primary tasks of all countries across the world.

The index R0 is widely used in the medical community to measure the infectivity of viruses, which refers to how many susceptible individuals will be infected by an infected person on average without any external intervention. Hellewell et al. develop a stochastic transmission model, parameterized to

\footnotetext{
${ }^{1}$ Source: https://www.adb.org/zh/news/covid-19-economic-impact-could-reach-8-8-trillion-globally-new-adb-report.
} 
the COVID-19 outbreak [2]. They find that when R0 is less than 1.5, the outbreak can be controlled without much intervention while much more strict measures must be taken to prevent the pandemic from spreading when R0 is more than 2.5. Even there is still no consensus on R0 of COVID-19 from the scientific community, most studies estimate that R0 is around $3[3,4]$, which indicates that the virus is highly infectious.

Despite some vaccines have put in the market, a big gap still exists between vaccine production capacity and the people to be vaccinated. Moreover, a partially effective vaccine can lead to more risky interactions, which may increase the probability that agents get infected $[5,6]$. Nanshan Zhong, a famous epidemiologist points out that prevention from the source is still the simplest but the most effective way. ${ }^{2}$ Cakir and Savas also show that a little increase in individual precautions can lead to "exponential progress" in pandemic prevention, which underscores the significant role of non-pharmaceutical interventions in pandemic prevention $[7,8]$.

Understanding of the virus infection mode is the very first thing of non-pharmaceutical interventions. Respiratory droplets and close contact are the main exposure routes of virus transmission. The transmission routes of aerosol and digestive tract are also possible in some extreme situations. People are likely to catch pneumonia unconsciously as long as they breathe in the environment with the existence of virus. The risk of infection becomes much higher in the crowded and closed spaces. What makes things worse is that the incubation period of the virus can be up to 14 days. The infectors are infectious even if they do not have any symptoms and thus an individual without any symptoms can also be dangerous. Nucleic acid testing is the most accurate method to identify infected individuals, it makes diagnosis by detecting virus specific nucleic acid sequence $[9,10]$. However, the nucleic acid testing requires expensive testing equipment and strict testing conditions, and also the individuals often need to make appointments in advance.

To effectively and efficiently prevent and control the virus, most governments have implemented decisive and resolute pandemic prevention and control measures. Most of the anti-pandemic policies aim to prevent the spread at domestic and the import from abroad. However, cross-country heterogeneities can be decisive [11]. Some countries have encountered great resistance in implementing these measures and have not achieved the desired results. Therefore, it is worth to assess the outbreak and relevant policies carefully.

Most studies assess the outbreaks by adopting the widely used SEIR dynamic model [10,12-16] which classifies all population into the following four categories: susceptible, exposed, infected, and recovered individuals. ${ }^{3}$ This model assumes that they can inter-convert according to certain parameters. This dynamic model is important for assessing the risk and predicting the peak of an outbreak. It also provides a reference for governments to formulate health policies. For instance, by analyzing data on the cases of COVID-19 in Wuhan during January 2020 and February 2020, Kucharski et al. point out that once there are more than four independent cases, there is more than 50\% chance the infection will establish in community level [10]. Eichenbaum et al. show that governments have to trade-off between higher risk of disease spreading and stronger economic downturn [15]. Acemoglu et al. find that a strict and long lockdown for the most vulnerable group is an effective access to reduce infections [16]. However, these works tend to emphasize "collective rationality" while ignored the importance of individual decision making in pandemic control and prevention.

\footnotetext{
${ }^{2}$ Source: http://k.sina.com.cn/article_1686546714_6486a91a020010dxr.html?from=international.

${ }^{3}$ In the SEIR model, a healthy but potentially at risk of infection individual is also referred to as a susceptible individual.
} 
In order to take individual or organization decision-making into account, some studies concentrate on using the game theory to solve the epidemiological issues [17-22]. Özkaya and İzgi use the pay-off matrix to analyze the effects of self-quarantine in the first wave of COVID-19 pandemic, and they find countries with higher benefits of self-quarantine (e.g. South Korea) are more likely to control the spread of the pandemic [17]. Reluga shows that social distancing could prevent disease transmission by reducing contact frequency, but people need to trade-off between social distancing and the risk of infection [18]. Social distancing is particularly useful when it is inexpensive and can delay the pandemic until a vaccine becomes widely available. Furthermore, Chen insists that the likelihood of eliminating an infectious disease through behavioral changes depends critically on the amount of information that individuals have access to [19]. Vilaca and Rodrigues attempt to analyze the process of influenza transmission using the ideas of repetitive games [20]. The above studies reveal the importance of individual behavioral decisions. However, these studies only consider very limited factors, nor do they recommend how to change the decisions.

In this study, we create a series of game models to investigate how some factors affect agents' best responses. In the first part of the study, we evaluate the factors that influence behavioral strategy of pandemic prevention based on a $2 * 2$ Bayesian game. We find that the high protective efficiency and risk exposure promote protection behavior, while the protection cost is in the opposite position. Next, we discuss the importance of information disclosure by relaxing the assumption that the protection cost is constant. In the second part, we use repeated games to analyze the best strategies to prevent the input of infectious cases. The results show that a punishment mechanism for airlines may reduce the imported cases, however we should also give airlines in low risk areas an opportunity to re-cooperate.

This study contributes to health policy and epidemiological literature in two ways. Methodologically, our game-theory-based approach reveals the individual-level decision making in pandemic prevention and control. Substantively, we introduce multiple factors to study the issue that is urgent and practical, which is crucial to improve our public health management system and cope with future public health emergencies.

\section{Individual and government behaviors}

\subsection{Adopting protective measures: A dominant strategy}

In order to analyze how individuals make protection decisions, let us conceive such a scenario: two agents, Ann and Bob are planning to meet and thus they have to decide whether they need to take protective measures (i.e. wearing masks, keeping social distance, etc.) or not in advance. To simplify the problem, we first assume that the protection cost $\mathrm{c}$ and the protective efficiency $1-\beta$ are fixed. Assume Ann and Bob know their own health status, but they can only estimate the unhealthy risk of the other agent based on the local prevalence rate and other relevant information. We denote the probability of Ann being an infector as $p$ in the perspective of Bob, and the probability of Bob as q in the perspective of Ann. In such a situation, these two agents have to make trade-offs between the risk of infection and the cost of protection including the monetary cost and the psychological cost.

By denoting the loss of catching the virus as $\alpha$, we assume that there exists the relation showing that $(1-\beta) \alpha>c$, which indicates that the benefits of taking measures can cover the costs. If one of the agents takes protective measures, the probability of virus transmission will reduce from 1 to $\beta$, and it goes to $\beta^{2}$ if they both take protective actions. $\beta$ and $\mathrm{q}$ are the possibilities, thus there exists that $0<\beta<1$, $0 \leqslant p, q \leqslant 1$. 
Table 1

Payoff matrix of adopting protective measures

\begin{tabular}{|c|c|c|}
\hline prob $=(1-p)(1-q)$ & protect & not \\
\hline protect & $-c,-c$ & $-c, 0$ \\
\hline not & $0,-c$ & 0,0 \\
\hline
\end{tabular}

(a) Both Ann and Bob are healthy

\begin{tabular}{|c|c|c|}
\hline$p r o b=p(1-q)$ & protect & not \\
\hline protect & $-\alpha-c,-\beta^{2} \alpha-c$ & $-\alpha-c,-\beta \alpha$ \\
\hline not & $-\alpha,-\beta \alpha-c$ & $-\alpha,-\alpha$ \\
\hline
\end{tabular}

(c) Only Ann is infected

\begin{tabular}{|c|c|c|}
\hline prob=(1-p)q & protect & not \\
\hline protect & $-\beta^{2} \alpha-c,-\alpha-c$ & $-\beta \alpha-c,-\alpha$ \\
\hline not & $-\beta \alpha,-\alpha-c$ & $-\alpha,-\alpha$ \\
\hline
\end{tabular}

(b) Only Bob is infected

\begin{tabular}{|c|c|c|}
\hline prob=pq & protect & not \\
\hline protect & $-\alpha-\mathrm{c},-\alpha-\mathrm{c}$ & $-\alpha-\mathrm{c},-\alpha$ \\
\hline not & $-\alpha,-\alpha-\mathrm{c}$ & $-\alpha,-\alpha$ \\
\hline
\end{tabular}

(d) Both Ann and Bob are infected

Table 2

Payoff matrix given Ann is healthy

Bob

\begin{tabular}{|c|c|c|c|}
\hline \multirow{4}{*}{ Ann } & prob=1-q & protect & not \\
\cline { 2 - 4 } & protect & $-\mathrm{c},-\mathrm{c}$ & $-\mathrm{c}, 0$ \\
\hline not & $0,-\mathrm{c}$ & 0,0 \\
\hline
\end{tabular}

(a) Bob is healthy

\begin{tabular}{|c|c|c|}
\hline prob $=\mathrm{q}$ & protect & not \\
\hline protect & $-\beta^{2} \alpha-\mathrm{c},-\alpha-\mathrm{c}$ & $-\beta \alpha-\mathrm{c},-\alpha$ \\
\hline not & $-\beta \alpha,-\alpha-\mathrm{c}$ & $-\alpha,-\alpha$ \\
\hline
\end{tabular}

(b) Bob is infected

Table 1 shows the payoff matrix of the game, which describes the benefits for two agents based on their health statuses. Without loss of generality, we can only consider how Ann adopts protective strategies. If Ann is infected, just like Table 1(c) and (d), the payment of Ann by adopting protective actions is:

$$
E A_{P}^{I}=-\alpha-c
$$

Otherwise, the payment of the infected Ann without adopting actions is:

$$
E A_{N}^{I}=-\alpha
$$

It is evident that the dominant strategy of Ann is taking no action if he/she is infected. ${ }^{4}$

Further, it is more significant to pay attention to how a healthy individual determines his/her protective strategy. The payoff matrix in Table 2 gives the case that Ann is healthy. If Bob is healthy as well, we can see that the payment of Ann adopting measures is $-c$; Otherwise, Ann bears no cost, and the payment is 0 . Under such a condition, taking no action should be the dominant strategy for Ann regardless of Bob's action. The interim Nash equilibrium of https://fanyi.baidu.com/-zh/en/javascript:void(0); This game is (not, not), and the payment is $(0,0) .^{5}$ However, if Bob is infected, as shown in Table 2(b), taking protection measures is expected to be the dominant strategy of Ann, since we know that Bob will not take any actions (just like what Ann will do when he/she is infected). In this situation, the interim Nash equilibrium is (protection, not), and the payment is $(-\beta \alpha-c,-\alpha)$.

Unfortunately, Ann does not know the health status of Bob. Therefore, he/she can only determine his/her strategy based on his/her estimation.

The expected payment of the healthy agent Ann by taking measures is:

$$
E A_{P}^{H}=-(1-q) c+q(-\beta \alpha-c)
$$

\footnotetext{
${ }^{4}$ A dominant strategy refers to the strategy optimal for the agent regardless of others' choice of strategy.

${ }^{5}$ Interim means an agent knows his/her own type but not the types of the other agents.
} 
The expected payment of the healthy agent Ann without taking actions is:

$$
E A_{N}^{H}=-q \alpha
$$

Assume Ann is a risk-neutral agent (i.e., always prefer higher expected return). She will take positive strategy if the excess payoff of taking protective measures (EPP) is greater than 0 :

$$
E P P^{H}=E A_{P}^{H}-E A_{N}^{H}=(1-\beta) \alpha q-c>0
$$

We can see that the protection decisions depend on the size of EPP in this game. Agents prefer to adopt positive strategies under the condition of higher EPP. Specifically, for given loss of catching the virus $\alpha$, three factors affect EPP according to expression (5):

(1) Protective efficiency $1-\beta$ is positively correlated with EPP.

(2) Protection cost $c$ is negatively correlated with EPP.

(3) Greater risk exposure q increases the value of EPP.

The above theoretical analyses enable us to understand the mechanism-based outcomes in different countries and regions by enforcing the control polices. For the regions with poor hygienic conditions, people suffer much more loss from this disaster mainly due to the low protective efficiency. In addition, in some areas advocating neoliberalism, much people face higher monetary costs and psychological costs. ${ }^{6}$ Furthermore, the decrease in the price of protective equipment due to the increasing capacity of medical equipment manufacturers also arouses the intensive attention of taking active protective measures.

It should be pointed out that the above analyses are conducted based on the assumption that their health status information is accessible to themselves. We can further expand the above model by assuming that the agents do not know the health status of anyone. Instead, they only know the probability distribution of the health status in both sides. In other words, agents need to make decisions under the circumstance of uncertain health.

In such a situation, the expected payment of Ann by taking measures is:

$$
E A_{P}=(1-p) E A_{p}^{H}+p E A_{P}^{I}=-(1-p)(1-q) c+(1-p) q(-\beta \alpha-c)+p(-\alpha-c)
$$

Otherwise, the expected payment without taking actions is:

$$
E A_{N}=(1-p) E A_{N}^{H}+p E A_{N}^{I}=-(1-p) q \alpha-p \alpha
$$

Similarly, a risk-neutral agent Ann is expected to take protective actions only if $E P P>0$, which yields:

$$
E P P=E A_{P}-E A_{N}=(1-p)(1-\beta) \alpha q-c>0
$$

Based on the model for uncertain health status, the conclusions are basically consistent with the predictions based on expression (5). In addition, expression (8) shows that people of higher possibility being healthy are more likely to take protective measures.

\footnotetext{
${ }^{6}$ Neoliberalism opposes government intervention in the market and restrictions on individual freedom; Therefore, the prices of medical materials are difficult to control. People also tend to doubt pandemic prevention recommendations from the government, which leads to higher psychological costs.
} 
Table 3

Payoff matrix of adopting protective measures (with continuous c)

\begin{tabular}{c|c|c|c|}
\multirow{4}{*}{ Ann } & prob=(1-q) & protect & not \\
\hline protect & $-c_{A},-c_{B}$ & $-c_{A}, 0$ \\
\hline not & $0,-c_{B}$ & 0,0 \\
\hline
\end{tabular}

(1) Bob is healthy

Bob

\begin{tabular}{|c|c|c|}
\hline prob $=\mathrm{q}$ & protect & not \\
\hline protect & $-\beta\left(\mathrm{c}_{\mathrm{A}}\right)^{2} \alpha-\mathrm{c}_{\mathrm{A}},-\alpha-\mathrm{c}_{\mathrm{B}}$ & $-\beta\left(\mathrm{c}_{\mathrm{A}}\right) \alpha-\mathrm{c}_{\mathrm{A}},-\alpha$ \\
\hline not & $-\beta\left(\mathrm{c}_{\mathrm{A}}\right) \alpha,-\alpha-\mathrm{c}_{\mathrm{B}}$ & $-\alpha,-\alpha$ \\
\hline
\end{tabular}

(2) Bob is infected

\subsection{Information revelation: A pareto improvement}

In the "adopting protective measures" game as illustrated in Section 2.1, the protection cost c is fixed. We can relax this assumption and regard $\mathrm{c}$ as a continuous variable on the basis of Table 2 . Thus, the protective efficiency $1-\beta$ is a function of cost $\mathrm{c}$ :

$$
\beta=\beta(c)
$$

It is easy to state that $\beta(0)=1$ and $\beta^{\prime}(c)<0$, where $\beta^{\prime}(c)$ is the first-order derivative of the function $\beta$ with respect to $c$. To ensure the existence and uniqueness of the equilibrium solution, we assume that $\beta^{\prime}(0)=-\infty$ and $\beta^{\prime \prime}(c)>0$, where $\beta^{\prime \prime}(c)$ is the second-order derivative of the function $\beta$ with respect to c. The significance of this assumption is that an agent is able to pay any amount of protection cost and reduce the risk of infection corresponding to the cost. Table 3 shows the payoff matrix. We still assume people know their health status and that Ann is healthy. We can prove that revealing the health status of an agent to the other agent is a Pareto improvement under the condition of information disclosure being costless. $^{7}$

Case 1. The health status of Bob is hidden to Ann

If the type of Bob is private information, Ann has to choose the optimal level of protection $\operatorname{cost} c_{A}^{*}$ based on his/her belief about $q$ in order to maximize the expected payoff $E A_{p}$, which can be mathematically expressed as:

$$
\max _{c_{A}} E A_{p}=-(1-q) c_{A}+q\left[-\beta(c) \alpha-c_{A}\right]
$$

By taking the first-order derivative of the function $E A_{p}$ with respect to $c_{A}$, yields:

$$
\left.\frac{\partial E A_{P}}{\partial c_{A}}\right|_{c_{A}=c_{A}^{*}}=-(1-q)+q\left[-\beta^{\prime}\left(c_{A}^{*}\right) \alpha-1\right]=-\alpha q \beta^{\prime}\left(c_{A}^{*}\right)-1=0
$$

Rearranging expression (11), yields:

$$
\beta^{\prime}\left(c_{A}^{*}\right)=-\frac{1}{\alpha q}<0
$$

Since $c_{A}=c_{A}^{*}$ is the optimal solution, $E A_{N}=\left.E A_{p}\right|_{c_{A}=0} \leqslant\left. E A_{p}\right|_{c_{A}=c_{A}^{*}}$, the optimal $E A_{p}$ should be no less than $E A_{N}$. Based on the calculation result, for positive $\alpha$ and $\mathrm{p}$, Ann is expected to choose a positive $\operatorname{cost} c_{A}^{*}$.

\footnotetext{
${ }^{7}$ Pareto improvement means to make at least one person better without making anyone worse.
} 
Table 4

Payment of Ann given Bob's type

\begin{tabular}{lcc}
\hline & Reveal & Not reveal \\
\hline Bob is healthy & 0 & $-c_{A}^{*}$ \\
Bob is infected & $-\beta\left(c_{A}^{* *}\right) \alpha-c_{A}^{* *}$ & $-\beta\left(c_{A}^{*}\right) \alpha-c_{A}^{*}$ \\
Expected payment & $-\beta\left(c_{A}^{* *}\right) \alpha q-c_{A}^{* *} q$ & $-\beta\left(c_{A}^{*}\right) \alpha q-c_{A}^{*}$ \\
\hline
\end{tabular}

Case 2. The type of Bob is revealed to Ann

If Bob is healthy, it will be the dominant strategy for Ann to take no action, and they both will get the payment of 0 . However, if Bob is infected, Ann will choose optimal $c_{A}=c_{A}^{* *}$ to maximizes his/her expected payoff $E A_{P}^{\prime}$ :

$$
\max _{c_{A}} E A_{p}^{\prime}=-\beta\left(c_{A}\right) \alpha-c_{A}
$$

By taking the first-order derivative of the function $E A_{P}^{\prime}$ with respect to $c_{A}$, yields:

$$
\left.\frac{\partial E A_{P}^{\prime}}{\partial c_{A}}\right|_{c_{A}=c_{A}^{* *}}=-\beta^{\prime}\left(c_{A}^{* *}\right) \alpha-1=-\alpha \beta^{\prime}\left(c_{A}^{* *}\right)-1=0
$$

Rearranging expression (14), yields:

$$
\beta^{\prime}\left(c_{A}^{* *}\right)=-\frac{1}{\alpha}
$$

Therefore, $c_{A}^{*}<c_{A}^{* *}$ due to the assumption that $\beta^{\prime \prime}(c)>0$.

The payment of Ann in case 1 and case 2 are presented in Table 4. Whether Bob is infected or not, the expected payment of Ann is always higher after revealing the type of Bob. Considering that revealing Bob's type does not influence the payment of him, EPP as well as the total expected payment after the disclosure increases:

$$
\left[-\beta\left(c_{A}^{* *}\right) \alpha q-c_{A}^{* *} q\right]-\left[-\beta\left(c_{A}^{*}\right) \alpha q-c_{A}^{*}\right]>0
$$

Thus, we conclude that information disclosure is a Pareto improvement in this game, which can help Ann choose his/her optimal protection level based on complete information. If the information of both agents is revealed, this game becomes a complete information game and the equilibrium becomes an ex-post Nash equilibrium. ${ }^{8}$ Either of the agents can make optimal decision based on all information in this game.

In the real world, however, revealing information is not cost-free. Local administration has to balance between the intensity level of information disclosure and its cost. If the intensity level of information is appropriate so that the extra benefit covers the cost, the disclosure increases the total payoff of society. Such measure is also a Pareto improvement if the disclosure cost is afforded by the susceptible individuals or society. Since the infected people have no incentive to pay the cost of information disclosure, we should not passively wait for the suspected patients to take the initiative to accept the test. Reducing the cost of information disclosure and additional punishment for concealing health status can help to encourage highrisk people to take the initiative to accept the nucleic acid test and "prevent from the source". Concerning that people are likely to overreact to information [23] or misunderstand the information [24-26], positive guidance and correct explanation are also indispensable.

\footnotetext{
${ }^{8}$ Ex-post means that the agent knows all agents' types.
} 
Table 5

Payoff matrix in the battle of pandemic import

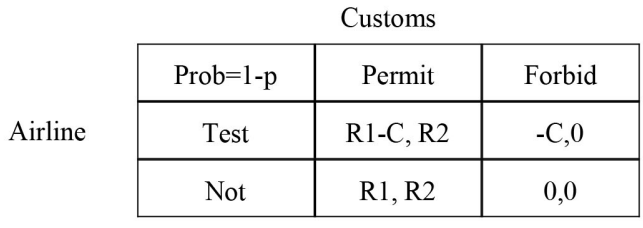

(a) No infector

\begin{tabular}{|c|c|c|}
\multicolumn{3}{|c}{ Customs } \\
\hline Prob=pq & Permit & Forbid \\
\hline Test & R1-C, R2 & -C,0 \\
\hline Not & R1, R2-L & 0,0 \\
\hline
\end{tabular}

(b) Exist infector and identified

\begin{tabular}{c|c|c|c|}
\multicolumn{1}{c|}{ Customs } \\
\cline { 2 - 4 } Airline & Prob=p(1-q) & Permit & Forbid \\
\cline { 2 - 4 } & Test & R1-C, R2-L & $-\mathrm{C}, 0$ \\
\cline { 2 - 4 } & Not & R1, R2-L & 0,0 \\
\hline
\end{tabular}

(c) Exist infector but not identified

\section{Customs quarantine strategy: Repeated games}

With the global spread of COVID-19, it has become the major task for customs to prevent the import of the virus. Some airlines are asked by local governments to guarantee their passengers to be healthy. Otherwise, the airlines will be punished, such as canceling flights. ${ }^{9}$ The game can be summarized as follows:

An airline decides whether to organize nucleic acid tests for passengers. Meanwhile, the customs decides whether to permit the entry or not. We assume that the probability of there existing infectors among the passengers is $\mathrm{p}$. The testing reliability is $\mathrm{q}$, which means that the possibility that all the infectors can be identified is $\mathrm{q} . \mathrm{R} 1$ and $\mathrm{R}_{2}$ are the revenues of the airline and local customs from opening-up respectively. The cost of confirming passenger's health condition is $C$ by assuming that the airline bears the costs, and the import of the virus causes loss $L$ to local government. In addition, we assume that $q R_{1}>C$ so that nucleic acid test is economically feasible and $R_{2}<L$. The payoff matrix is shown in Table 5 .

If this is a single-stage game, the equilibrium is obvious. "Not test" will be the dominant strategy since it brings more payoff in all cases. The customs may suffer a loss because of virus introduction.

However, if we regard this case as repeated games [17,20], cooperation becomes possible. Noticing that (test, permit) is a win-win outcome compared with (not, forbid), cooperation becomes possible. The problem that the customs faces is to ensure that the airline does not deviate from cooperation. Assume that the airline cares about the future just as much as the present, but with probability of $1-\beta$ that the pandemic will be over in any given round. ${ }^{10}$ Two strategies of customs will be considered as following:

Case 1: Trigger.

Start out permitting, and once an unidentified infector is found and cause losses, the customs will play "forbid" forever. Since the payoff outcome of a stage is given before next stage begins, the airline can predict what actions the customs will play in next stage. Thus, we can eliminate the outcome of (test, not).

\footnotetext{
${ }^{9}$ Source: http://www.caac.gov.cn/PHONE/XXGK_17/XXGK/TZTG/202012/t20201216_205607.html.

${ }^{10}$ This is equivalent to the situation that the pandemic continues, but the discount rate of the company is $1-\beta$.
} 


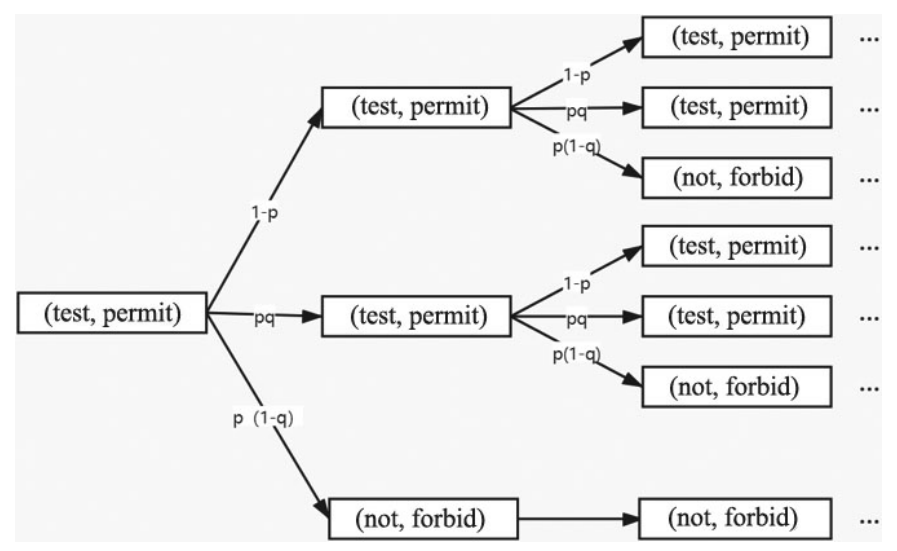

Fig. 1. Outcomes: the cooperative airline vs. the customs of trigger.

Given the action strategy of the customs, we can predict what the airline will do. If the airline plays "test" when the customs plays "permit", the performances of the outcomes can be shown in Fig. 1. There are 3 possible outcomes:

(1) There is no infector among the passengers and the customs will play "permit" in the next stage, the possibility is $1-p$, as shown in Table 5(a).

(2) There exist infectors among the passengers and they are all identified, the custom will play "permit" in the next stage, the possibility is pq, as shown in Table 5(b).

(3) There exist infectors among the passengers but some of them can not be identified, the customs will suffer the loss L and play "forbid" forever, the possibility is $p(1-q)$, as shown in Table 5(c).

Notice that the sub game with the beginning node of (test, permit) is the same as the original game. The PV (present value) of the airline to cooperate is:

$$
P V A_{c}^{1}=\left(R_{1}-C\right)+\beta(1-p) P V A_{c}^{1}+\beta p q P V A_{c}^{1}+p(1-q) * 0
$$

Rearranging expression (17), yields:

$$
P V A_{c}^{1}=\frac{R_{1}-C}{1-\beta+\beta p-\beta p q}
$$

We can calculate the PV of the customs as:

$$
P V C_{c}^{1}=(1-p)\left(R_{2}+\beta P V C_{c}^{1}\right)+p q\left(R_{2}+\beta P V C_{c}^{1}\right)+p(1-q)\left(R_{2}-L\right)
$$

Rearranging expression (19), yields:

$$
P V C_{c}^{1}=\frac{R_{2}-p(1-q) L}{1-\beta+\beta p-\beta p q}
$$

Similarly, if the aviation company plays "not" when the customs plays "permit", the performances of the outcomes can be shown in Fig. 2. The sub game with the beginning node of (not, permit) is the same as the original game. The $\mathrm{PV}$ of the airline to defect is:

$$
P V A_{d}^{1}=R_{1}+\beta(1-p) P V A_{d}^{1}+p q * 0+p(1-q) * 0
$$

Rearranging expression (21), yields:

$$
P V A_{d}^{1}=\frac{R_{1}}{1-\beta+\beta p}
$$




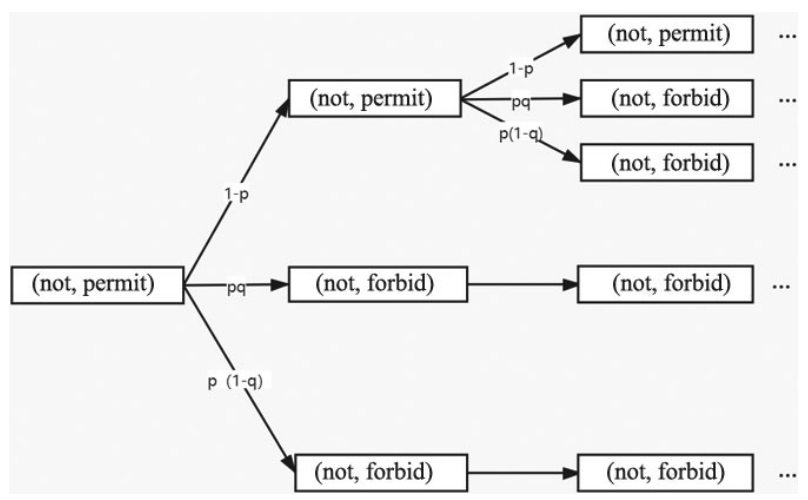

Fig. 2. Outcomes: the airline of defection vs. the customs of trigger.

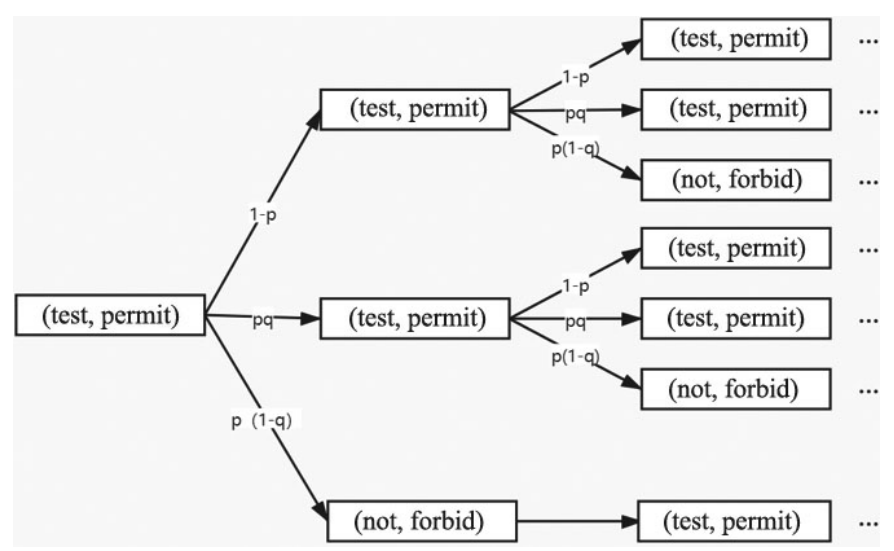

Fig. 3. Outcomes: the cooperative airline vs. the customs of tit for tat.

The PV of the customs in this situation is:

$$
P V C_{d}^{1}=(1-p)\left(R_{2}+\beta P V C_{d}^{1}\right)+p q\left(R_{2}-L\right)+p(1-q)\left(R_{2}-L\right)
$$

Rearranging expression (29), yields:

$$
P V C_{d}^{1}=\frac{R_{2}-p L}{1-\beta+\beta p}
$$

When $P V A_{c}^{1}>P V A_{d}^{1}$, it is no good for the airline to defect, which means:

$$
\beta>\frac{C}{C-p C+p q R_{1}}
$$

Case 2: Tit for tat.

Start out permitting, and once an unidentified infector is found and cause losses, the customs will play "forbid" in the next stage, then go back to "permit". Instead of playing "forbid" forever, the customs only punish the airline for one-stage. It is a strategy with both punishment and forgiveness.

If the airline plays "test" when the customs plays "permit", the performances of the outcomes is shown in Fig. 3. As in the first case, the sub game with the beginning node of (test, permit) is the same as the original game. We can calculate the PV of the airline and the customs in such a situation:

$$
P V A_{c}^{2}=\left(R_{1}-C\right)+\beta(1-p) P V A_{c}^{2}+\beta p q P V A_{c}^{2}+\beta p(1-q)\left(0+\beta P V A_{c}^{2}\right)
$$




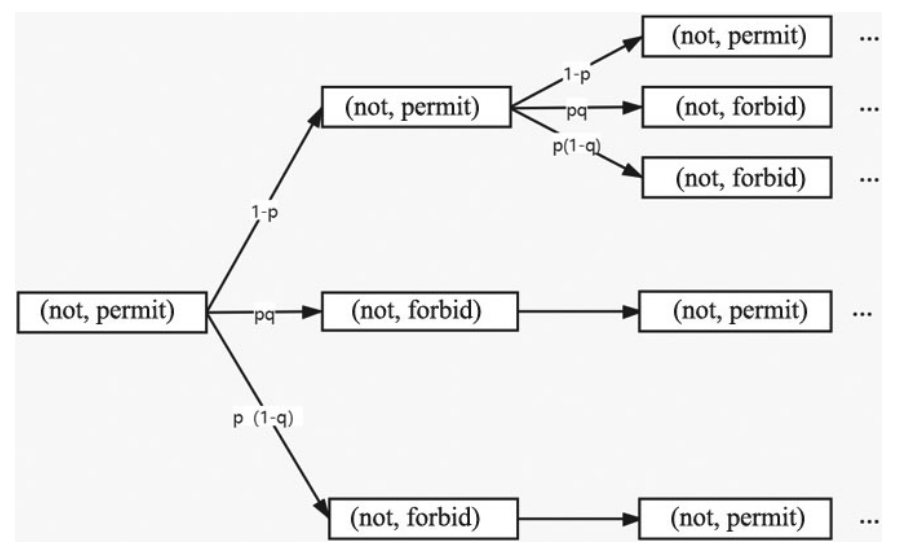

Fig. 4. Outcomes: The airline of defection vs. the customs of tit for tat.

Rearranging expression (32), yields:

$$
\begin{aligned}
P V A_{c}^{2} & =\frac{R_{1}-C}{(1-\beta)(1+\beta p-\beta p q)} \\
P V C_{c}^{2} & =(1-p)\left(R_{2}+\beta P V C_{c}^{2}\right)+p q\left(R_{2}+\beta P V C_{c}^{2}\right)+p(1-q)\left(R_{2}-L+\beta^{2} P V C_{c}^{2}\right)
\end{aligned}
$$

Rearranging expression (28), yields:

$$
P V C_{c}^{2}=\frac{R_{2}-p(1-q) L}{(1-\beta)(1+\beta p-\beta p q)}
$$

If the airline plays "not" when the customs play "permit", the performances of the outcomes can be shown in Fig. 4. The PV of the airlines and the customs are:

$$
P V A_{d}^{2}=R_{1}+\beta(1-p) P V A_{d}^{2}+\beta p q\left(0+\beta P V A_{d}^{2}\right)+\beta p(1-q)\left(0+\beta P V A_{d}^{2}\right)
$$

Rearranging expression (30), yields:

$$
\begin{aligned}
P V A_{d}^{2} & =\frac{R_{1}}{(1-\beta)(1+\beta p)} \\
P V C_{d}^{2} & =(1-p)\left(R_{2}+\beta P V C_{d}^{2}\right)+p q\left(R_{2}-L+\beta^{2} P V C_{d}^{2}\right)+p(1-q)\left(R_{2}-L+\beta^{2} P V C_{d}^{2}\right)
\end{aligned}
$$

Rearranging expression (32), yields:

$$
P V C_{d}^{2}=\frac{R_{2}-p L}{(1-\beta)(1+\beta p)}
$$

The airline does not defect on the condition that $P V A_{c}^{2}>P V A_{d}^{2}$, which means:

$$
\beta>\frac{C}{p q R_{1}-p C}
$$

Compared expression (34) with expression (31), we can see that the limit to $\beta$ is more strict in the latter case. Moreover, we find that tit-for-tat strategy is better than trigger strategy if the infection rate $\mathrm{p}$ is rather low. For the airline, we find that $P V A_{c}^{1}<P V A_{c}^{2}$ and $P V A_{d}^{1}<P V A_{d}^{2}$, which means that tit-for-tat strategy is always preferred by the airline. However, the customs prefers tit for tat only if $p<\frac{R_{2}}{(1-q) L}$ for defection airlines or $p<\frac{R_{2}}{L}$ for cooperative airlines. 
The model shows the application of repeated game theory in the field of pandemic prevention and control. First, consistent with folk theorem [27], if the discount rate $\beta$ is high enough, we can achieve the cooperation that is impossible in single-stage games. However, when $\beta$ is very low, the cooperation is hard to achieve, and the airline will always play "not test". In both cases, the threshold of $\beta$ is positively correlated with the cost $C$, and it is negatively correlated with the revenue of airlines $R_{1}$ and the testing reliability q. ${ }^{11}$ Aviation companies with lower profit margin (e.g. low-cost airlines) are more likely to cancel their flights or require passengers to bear the cost of testing.

In addition, the tit-for-tat strategy requires higher $\beta$ compared with that of trigger, which means the airline is more likely to defect. However, when $\mathrm{p}$ is very low, tit for tat brings more benefits to both sides. This is because the tit-for-tat strategy allows forgiveness for airlines that have temporarily betrayed or missed diagnosis due to accidents, and achieves more cooperation. This is consistent with the idea that repeated interaction and effective communication may overcome the influence of self-interest behavior and asymmetric information and bring about the result of long-term cooperation [28]. In order to achieve such desirable outcomes, appropriate forgiveness is indispensable.

\section{Summary and conclusions}

Based on the game theory, this study provides the theoretical support for government policies to prevent and control COVID-19, and explains why similar policies achieve very different effects in different countries and regions.

In the first model, we review how people adopt protection strategies in a $2 * 2$ Bayesian game. It shows that the protection cost, the protective efficiency and the risk exposure are the key factors that affect decision-making of healthy individuals. In terms of health policy, it would be useful to encourage the construction of new mask production lines and increase the supply of other protection materials. In addition, advocating correct hygiene manners in social activities is also helpful to improve protective efficiency and promote positive protection strategies. In contrast, a severe outbreak of COVID-19 may occur due to the lack of protective measures in some areas where neoliberalism prevails or basic hygienic conditions are difficult to guarantee.

Furthermore, by relaxing the assumption that protection cost is constant, the model underscores the importance of the disclosure of health information. Information disclosure, such as publicizing the local vaccination rate and the itinerary of infectors, increase social welfare by influencing individual decisionmaking. According to the result, measures aim to reduce the cost of information disclosure and promote information disclosure is of great significance to improve social welfare and limit the spread of the pandemic. In China, "Trip Code" enables residents to display where they have been recently according to GPS data, further reducing the cost of disclosing information considerably. The European Union's digital vaccine passport also allows low-risk international travelers to provide low-cost health signals. These methods have effectively promoted information disclosure and restricted the spread of the pandemic with the help of digital technologies. Facing the risk of future pandemic transmission, we should encourage further innovation in improving the detection efficiency and reducing the cost of information disclosure.

In the second model, we discuss the best strategy to prevent the import of the virus by developing repeated games between local customs and airlines. Based on the uncertainty of detection efficiency and

\footnotetext{
${ }^{11}$ Airlines' threshold is defined here as the lowest discount rate $\beta$ at which the airlines play "test" to maximize the present value.
} 
technology, we find it meaningful to set up a punishment mechanism for aviation companies to release the pressure of customs. However, the mechanism works only if the discount rate of the airline is greater than the threshold. The threshold is positively correlated with the detection cost, and it is negatively correlated with the revenue of the airline and the testing reliability. We also benefit from establishing a credit mechanism to give airlines that deviate from cooperation an opportunity to re-cooperate, especially for those in low risk areas.

The result of this study underscores the significance of taking into account individual and organizational decision-making in policy making. Health policy design based on users' and airlines' decisions is more conducive to the implementation of policies and preventing the spread of the pandemic. Measuring these interventions is also crucial to improve our public health management system and better respond to future public health emergencies.

While this study highlights the reference value of game theory approach in public health policy making, there are several limitations. First, this study is based on a game analysis that is not directly related to the field relationship, but to the outcome relationship. Although we have proposed several criteria to analyze individual and organizational decision-making, they are still limited compared with the reality. Future research should go beyond our theoretical analysis by combining with behavioral data based on real residents' decision-making. Second, our study mainly concerns the general factors affecting behavior in pandemic prevention and control. Some direct monetary interventions, such as fines and rewards, are not included in this study. Third, the models in our study are two-player games. It may also be interesting to discuss the cases of multi-player games about the above issues.

\section{References}

[1] United Nations, Shared responsibility, global solidarity: Responding to the socio-economic impacts of COVID-19, United Nations (2020).

[2] J. Hellewell, S. Abbott, A. Gimma, I.N. Bosse, C.I. Jarvis, T.W. Russel, J. Munday, A.J. Kucharski and J. Edmunds, Feasibility of controlling COVID-19 outbreaks by isolation of cases and contacts, The Lancet Global Health 8(4) (2020), e488-e496.

[3] Y. Liu, A.A. Gayle, W.S. Annelies and J. Rocklöv, The reproductive number of COVID-19 is higher compared to SARS coronavirus, Journal of Travel Medicine 27(2) (2020), 1-4.

[4] A.J. Kucharski, T.W. Russel, C. Diamond, Y. Liu, J. Edmunds, S. Funk and R.M. Eggo, Early dynamics of transmission and control of COVID-19: A mathematical modelling study, Lancet Infect Dis (2020) published online March 11.

[5] E. Talamàs and R. Vohra, Free and perfectly safe but only partially effective vaccines can harm everyone, Games and Economic Behavior 122 (2020), 277-289.

[6] A. Al-Hazmi, I. Gosadi, A. Somily, S. Alsubaie and A. Bin Saeed, Knowledge, attitude and practice of secondary schools and university students toward middle east respiratory syndrome epidemic in Saudi Arabia: A cross-sectional study, Saudi Journal of Biological Sciences 25(3) (2018), 572-577.

[7] Z. Cakir and H.B. Savas, A mathematical modelling for the COVID-19 pandemic in Iran, Ortadogu Tip Dergisi 12(2) (2020), 206-210.

[8] B. Tu and K. Liu, Community prevention and control mechanism of public health emergency - based on the perspective of social force participation in China, Advances in Industrial Engineering and Management 9(1) (2020), 47-50.

[9] C. Huang, Y. Wang and X. Li, Clinical features of patients infected with 2019 novel coronavirus in Wuhan, China, The Lancet 395 (2020), 10223.

[10] P. Zhou, X.L. Yang, X.G. Wang, B. Hu, L. Zhang, W. Zhang, H.R. Si, Y. Zhu, B. Li and C.L. Huang, A pneumonia outbreak associated with a new coronavirus of probable bat origin, Nature 579(7798) (2020), 270-273.

[11] D.E. Bloom, M. Kuhn and K. Prettner, Modern infectious diseases: Macroeconomic impacts and policy responses, Journal of Economic Literature (2020), 27757.

[12] A. Radulescu and K. Cavanagh, Management strategies in a SEIR model of COVID-19 community spread, Scientific Reports 10(1) (2020), 21256.

[13] A. Lipton and L.D.P. Marcos, Mitigation strategies for COVID-19: Lessons from the K-SEIR model, Social Science Electronic Publishing (2020). 
[14] S. Mwalili, M. Kimathi, V. Ojiambo, D. Gathungu and R.W. Mbogo, SEIR model for COVID-19 dynamics incorporating the environment and social distancing, Nature Public Health Emergency Collection 13(1) (2020), 352.

[15] M.S. Eichenbaum, S. Rebelo and M. Trabandt, The macroeconomics of epidemics, NBER Working Paper No 26882, National Bureau of Economic Research 2020a, Cambridge, MA, USA.

[16] D. Acemoglu, V. Chernozhukov, I. Werning and M.D. Whinston, Optimal targeted lockdowns in a multi-group SIR model, NBER Working Papers (2020).

[17] M. Özkaya and B. İzgi, Effects of the quarantine on the individuals' risk of COVID-19 infection: Game theoretical approach, Alexandria Engineering Journal 60(4) (2021), 4157-4165.

[18] T.C. Reluga, Game theory of social distancing in response to an epidemic, Plos Computational Biology 6(5) (2010), e1000793.

[19] F.H. Chen, Modeling the effect of information quality on risk behavior change and the transmission of infectious disease, Mathematical Bioences 217(2) (2008), 125-133.

[20] X. Vilaca and L. Rodrigues, On the effectiveness of punishments in a repeated epidemic dissemination game, in: T. Higashino, Y. Katayama, T. Masuzawa, M. Potop-Butucaru and M. Yamashita, eds., Springer, Cham, Berlin, 2013, pp. 206-220.

[21] Y.Y. Feng and Z.F. Wang, Development mode of circular economy industrial cluster based on game theory, International Journal of Computer Applications in Technology 61(1-2) (2019), 47-53.

[22] A. Caparros and M. Finus, The corona-pandemic: A game theoretical perspective on regional and global governance, Environmental and Resource Economics 76 (2020), 913-927.

[23] Y. Zhang and R. Zhou, Promoting social distancing and preventing panic buying during the epidemic of COVID-19: The contributions of people's psychological and behavioural factors, Journal of Public Health (4) (2021), 615.

[24] C.H. Basch, Z. Meleo-Erwin, J. Fera, C. Jaime and C.E. Basch, A global pandemic in the time of viral memes: COVID-19 vaccine misinformation and disinformation on TikTok, Human Vaccines and Immunotherapeutics, 2021, pp. 1-5.

[25] J.L. Bonnet and S. Sellers, The COVID-19 misinformation challenge: an asynchronous approach to information literacy, Internet Reference Services Quarterly (3) (2021), 1-8.

[26] A.P. Worrall, M.J. Connolly, A. O'Neill, M. O'Doherty, K.P. Thornton, C. McNally, S.J. McConkey and E. de Barr, Online readability of COVID-19 health information: A comparison between four English speaking countries, BMC Public Health 20(1) (2020), 1635.

[27] D. Fudenberg and E. Maskin, The folk theorem in repeated games with discounting or with incomplete information, Econometrica 54(3) (1986), 533-554.

[28] E.J. Toikka, Efficiency in games with markovian private information, Econometrica 81(5) (2013), 1887-1934. 